\title{
Macroscopic analysis of human masseter compartments assessed by magnetic resonance imaging
}

\author{
Cioffi, I ; Gallo, L M ; Palla, S ; Erni, S ; Farella, M
}

\begin{abstract}
The human masseter is divided into compartments by aponeuroses. So far, the qualitative and quantitative features of these compartments and their aponeuroses have been scarcely investigated. This study investigated the three-dimensional compartmentalization of the masseter muscle and tested the hypothesis that aponeurosis content varies systematically across different masseter subportions as well as between genders. The right masseter of 14 healthy participants was scanned by magnetic resonance, and the outlines of muscle and aponeuroses were segmented and rendered in three dimensions by AMIRA software. The internal architecture of the masseter muscle varied markedly across individuals, with respect to the number, shape and location of the compartments delimited by aponeuroses. Aponeuroses were widely represented inside the masseter, amounting to $7.1 \pm 2.1 \%$ of its volume. The aponeurosis content varied systematically across masseter subvolumes and did not differ between genders after adjusting for body height and weight.
\end{abstract}

DOI: https://doi.org/10.1159/000329503

Posted at the Zurich Open Repository and Archive, University of Zurich

ZORA URL: https://doi.org/10.5167/uzh-54870

Journal Article

Published Version

Originally published at:

Cioffi, I; Gallo, L M; Palla, S; Erni, S; Farella, M (2012). Macroscopic analysis of human masseter compartments assessed by magnetic resonance imaging. Cells Tissues Organs, 195(5):465-472.

DOI: https://doi.org/10.1159/000329503 


\title{
Macroscopic Analysis of Human Masseter Compartments Assessed by Magnetic Resonance Imaging
}

\author{
lacopo Cioffia, b Luigi Maria Gallo ${ }^{a}$ Sandro Palla ${ }^{a}$ Stefan Erni ${ }^{a}$ \\ Mauro Farella ${ }^{b, c}$ \\ ${ }^{a}$ Clinic for Masticatory Disorders, Removable Prosthodontics, and Special Care Dentistry, University of Zürich, \\ Zürich, Switzerland; b Discipline of Orthodontics, Department of Dental, Oral, and Maxillo-Facial Sciences, \\ Federico II University of Naples, Naples, Italy; ' $D$ iscipline of Orthodontics, Department of Oral Sciences, \\ University of Otago, Dunedin, New Zealand
}

\section{Key Words}

Masseter · Aponeurosis • Magnetic resonance imaging • Muscle compartments

\begin{abstract}
The human masseter is divided into compartments by aponeuroses. So far, the qualitative and quantitative features of these compartments and their aponeuroses have been scarcely investigated. This study investigated the three-dimensional compartmentalization of the masseter muscle and tested the hypothesis that aponeurosis content varies systematically across different masseter subportions as well as between genders. The right masseter of 14 healthy participants was scanned by magnetic resonance, and the outlines of muscle and aponeuroses were segmented and rendered in three dimensions by AMIRA software. The internal architecture of the masseter muscle varied markedly across individuals, with respect to the number, shape and location of the compartments delimited by aponeuroses. Aponeuroses were widely represented inside the masseter, amounting to $7.1 \pm 2.1 \%$ of its volume. The aponeurosis content varied systematically across masseter subvolumes and did not differ between genders after adjusting for body height and weight.

Copyright $\odot 2011$ S. Karger AG, Basel
\end{abstract}

\section{Introduction}

The masseter muscle plays a major role in all jaw functional activities such as chewing, biting, drinking, swallowing and speaking [Farella et al., 2008]. Moreover, it is also strongly active during nonfunctional activities such as tooth clenching and grinding that are risk factors for masticatory muscle pain [Huang et al., 2002; Michelotti et al., 2010].

Studies performed on the trapezius muscle indicate that subjects with stereotypical contraction patterns have an increased risk of developing muscle pain [Falla and Farina, 2008; Falla et al., 2010; Farina et al., 2008]. Furthermore, stereotypical contractions of specific muscle subportions have been implicated in the pathogenesis of muscle pain, especially in work-related musculoskeletal pain [Sjøgaard and Søgaard, 1998]. The Cinderella hy-

\begin{tabular}{ll} 
Abbreviations used in this paper \\
\hline BMI & body mass index \\
EMG & electromyographic \\
MRI & magnetic resonance imaging \\
SD & standard deviation
\end{tabular}


pothesis, for instance, postulates that some motor units containing type I fibers (Cinderella motor units) are continuously activated in a stereotypic fashion during prolonged low-level motor tasks, and may therefore become overloaded and damaged, leading to focal inflammation and, eventually, to nociceptor sensitization and muscle pain [Hägg et al., 1991].

The human masseter is subdivided into different compartments; it has motor unit territories confined within the muscle's internal aponeuroses [Tonndorf et al., 1994] and is functionally heterogeneous [Blanksma et al., 1992; McMillan and Hannam, 1992; McMillan, 1993; Blanksma and Van Eijden, 1995; Schindler et al., 2005]. This means that during jaw functioning, muscle subportions can be differentially activated, i.e. some parts of the masseter muscle are more active than others [Palla and Farella, 2010]. Also, during a low-level prolonged contraction the area with the highest EMG amplitude, that corresponds to the area with the highest contraction level, may or may not gradually move in a sagittal direction throughout the contraction task [unpublished observations], showing that in some individuals the contraction pattern is less constant (i.e. less stereotypical) than in others. Lastly, the physiological properties of the masseter muscle are consistent with anatomical and morphological findings such as the focal distribution of the territories of motor units in this muscle [Van Eijden and Turkawski, 2001], the great variability in fiber type composition across various muscle portions [Eriksson and Thornell, 1983], the regional differences in blood perfusion and nerve supply [Niioka et al., 2009], and muscle compartmentalization [Widmer et al., 2007]. Therefore, in order to further investigate the role of stereotypical contractions in the pathophysiology of masticatory muscle pain, it is important to increase our knowledge of the internal architecture of the jaw muscles.

Previous anatomical studies [Schumacher, 1961; McMillan and Hannam, 1992; Gaudy et al., 2000] indicated that the human masseter muscle shows a typical pennate architecture, which includes thick and robust aponeurotic layers delimiting discrete subvolumes (i.e. muscle compartments). This internal organization represents an anatomical counterpart of functional heterogeneity and may help explaining the large forces produced by the masseter muscle in various directions of space [Koolstra and Van Eijden, 1992]. To date, internal aponeuroses of the masseter muscles have been scarcely investigated and little information is available regarding their size, location, gender-related differences and specific three-dimensional arrangements.
Advances in magnetic resonance imaging (MRI) have increased our potential to investigate the musculoskeletal system in detail [Augat and Eckstein, 2008], including the masticatory muscles [Gaudy et al., 2000; Ng et al., 2006, 2008]. The aponeuroses of the masseter muscle, for instance, can be rendered in three dimensions and assessed by software thus opening the possibility to study the compartmentalization of this muscle.

Hence the aims of this study were to describe the internal architecture of the masseter muscle in healthy young volunteers using in vivo MRI in order to investigate the three-dimensional arrangement of muscle compartments and their aponeuroses, and to determine whether muscle and aponeurosis volumes differ between genders. The specific research questions to be addressed were: are the masseter muscle compartments arranged according to a consistent three-dimensional pattern? Is there any difference in aponeurosis content between genders? These questions are relevant in order to investigate whether selected parts of the masseter muscle are stereotypically contracted in some individuals putting them at higher risk of developing masticatory muscle pain.

\section{Materials and Methods}

A convenience sample of 14 healthy participants $(7$ males, mean age $\pm \mathrm{SD}=26.3 \pm 2.6$ years; 7 females $=25.8 \pm 2.5$ years; total sample: $26.0 \pm 2.5$ years) was recruited among students and staff members of the Clinic for Masticatory Disorders, Removable Prosthodontics, and Special Care Dentistry, University of Zürich, according to the following inclusion criteria: adult age ( $>18$ years) and willingness to participate in the experiment. The following conditions were considered as exclusion criteria: self-report of temporomandibular joint or muscle pain; painful or limited jaw movements (i.e. active maximum mouth opening $<40 \mathrm{~mm}$ (including overbite), protrusion and laterotrusion $<7 \mathrm{~mm}$; difference between active and passive opening $\leq 2 \mathrm{~mm}$ ); tenderness to digital palpation of the masseter and temporal muscles as well as of the temporomandibular joint area; crepitation and clicking sounds of the temporomandibular joints; dental prostheses; absence of more than 2 teeth (with the exception of third molars); neurological disorders; overjet $<0$ or $>7 \mathrm{~mm}$, and unilateral or bilateral cross-bite.

All participants were carefully informed about the procedures and underwent a clinical examination to exclude the presence of signs of temporomandibular and muscle disorders, i.e. of a myoarthropathy of the masticatory system. The examination was performed according to the standard protocol described in the Research Diagnostic Criteria for Temporomandibular Disorders [Dworkin and Le Resche, 1992]. Differently to this standard protocol, only the masseter and temporalis muscle were palpated. In addition, the examination also included the measurement of overjet and overbite as well as the assessment of cross-bite.

Body weight, body height and body mass index (BMI) of each participant were assessed using conventional methods. 


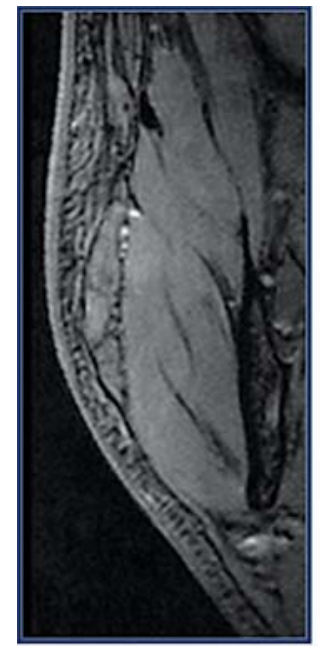

$\mathbf{a}$
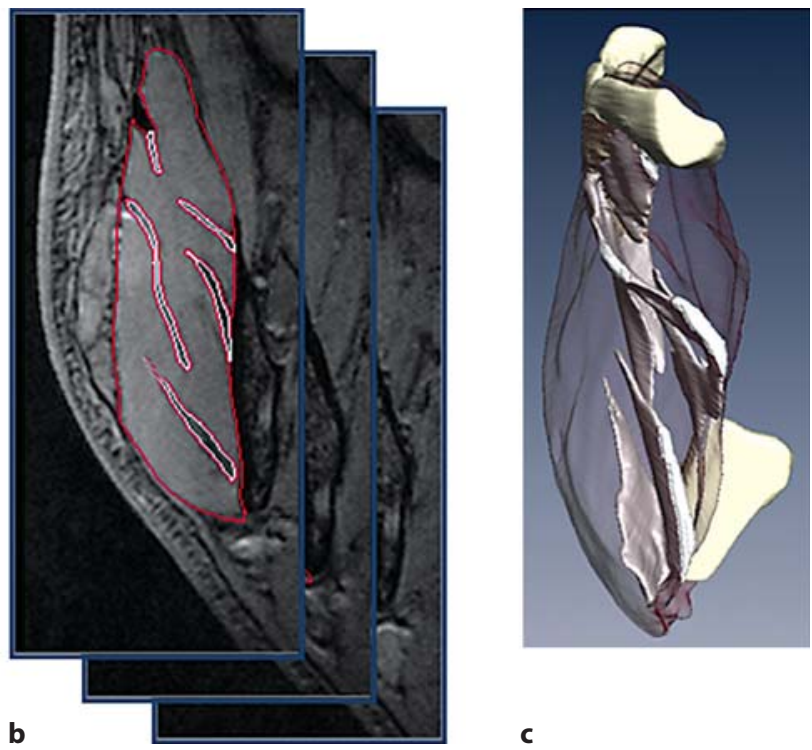

C

Fig. 1. Schematic representation of the method used for segmentation and three-dimensional rendering of masseter aponeuroses. a Cross-sectional scan of one human masseter. b Segmentation of muscle boundaries and aponeuroses on consecutive scans. c Three-dimensional rendering by software. Note: masseter aponeuroses are identifiable as low-intensity signals inside the muscle belly.

\section{Magnetic Resonance Imaging}

In order to insure that the subject kept his/her jaw firm and in a standard position throughout the magnetic resonance scans, a custom-made check-bite was constructed by applying a layer of vinyl polysiloxane (Blu-Mousse, Parkell Inc., N.Y., USA) from the right lower canine to the right lower first molar, and by asking the subject to close his/her mouth in the intercuspal position.

MRI scans of the masseter muscles were taken 3-5 days after the clinical examination with the subject positioned in the supine position, so that the Frankfurt plane was perpendicular to the scan table. A $10-\mathrm{cm}$ diameter coil, originally developed for temporomandibular joint recordings, was placed over the right masseter. The coil was centred on the mandibular ramus in slight contact with facial skin, thus increasing head stability. During the recordings, subjects were instructed to hold the check-bite in place while keeping teeth in contact with minimum contraction effort.

Coronal scans of the right masseter muscle were obtained by means of a 1.5-tesla scanner (Gyroscan Intera, Philips Medical Systems, Best, The Netherlands) using T1-weighted sequences within a $130 \times 130 \mathrm{~mm}$ field of view and a two-dimensional gradient echo-type sequence. Slice thickness was set at $2 \mathrm{~mm}$, and pixel spacing at $0.51 \mathrm{~mm}$. The interslice gap was $2 \mathrm{~mm}$. Repetition and echo times were 500 and $7.8 \mathrm{~ms}$, respectively. Voxel size was $0.51 \times 1.99$ $\times 0.51 \mathrm{~mm}$, the acquisition matrix was $256 \times 230$ pixels. The complete scan sequence obtained for each masseter included 28 coronal slices that were stored as Digital Imaging and Communications in Medicine files for postprocessing. The protocol was approved by the Local Ethics Committee (reference No. StV 08/02) and written informed consent was obtained from all participants.

\section{Image Analysis}

Individual scans of the right masseter muscle were imported and analyzed by AMIRA (5.2 Visage Imaging software, Berlin, Germany). Two-millimeer-thick slices are thin enough to reconstruct the inner architecture of the masseter muscle. However, in order to obtain smooth three-dimensionally rendered volumes, the original voxel size was reduced to $0.169 \times 0.169 \times 0.67 \mathrm{~mm}$ by image interpolation. Thereafter, the images of each masseter were displayed as a series of 84 contiguous coronal images at an interval of $0.67 \mathrm{~mm}$.

The outlines of the masseter muscle, muscle aponeuroses, zygomatic arch and mandibular corpus were initially identified by software using an automatic built-in segmentation tool and then fine-tuned by manual contour tracing by a trained examiner (I.C.). The aponeuroses of the masseter muscle could be clearly depicted as low-intensity signals inside muscle tissues (fig. 1). The accuracy of segmentation was verified across all the coronal, sagittal and axial slices. When the outlines of an aponeurosis were not distinct, these were double-checked by a second examiner (M.F.) and discussed until a consensus was reached. The volumes of the segmented muscles and aponeurosis tissue were calculated via a software algorithm and expressed graphically (fig. 2).

After segmentation, a set of points and reference planes (fig. 2a) was constructed in order to reorient the graphic data set and to subdivide each masseter into eight subvolumes (regions of interest). Two points were first selected: the zygomatic point, as the most inferior point midway along the zygomatic arch, and the gonion point as the point where the lower border of the mandible curves upward (fig. 2a). The original coronal MR slices were reori- 


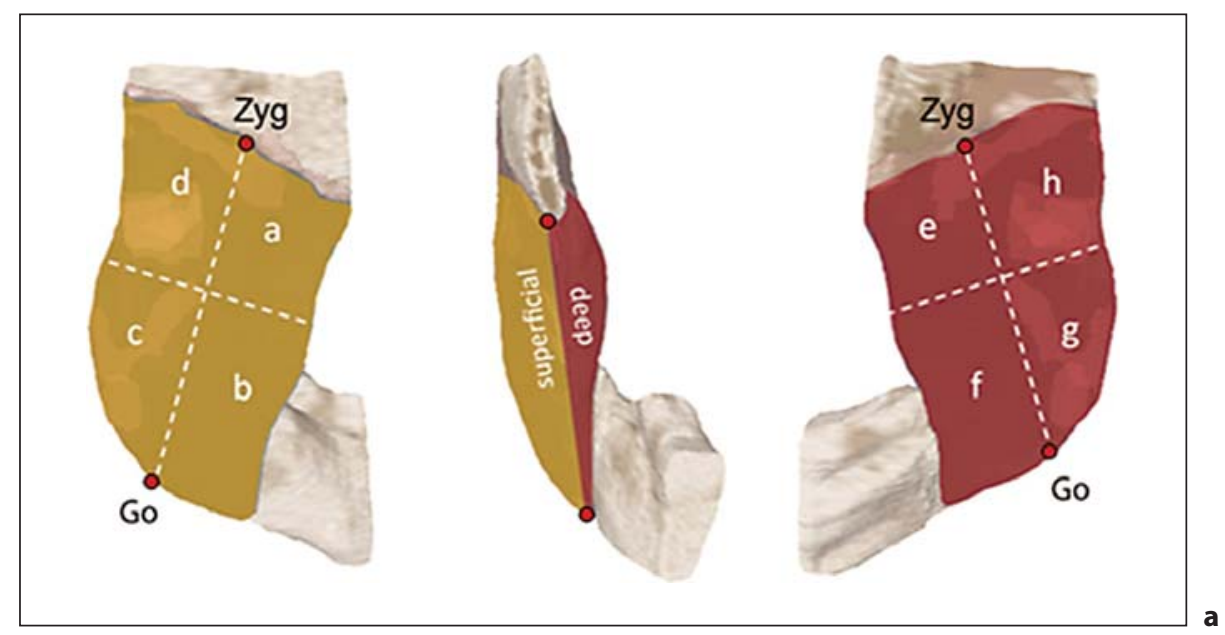

\begin{tabular}{|llll|}
\hline Subportion & $\begin{array}{l}\text { Aponeurosis relative } \\
\text { content } \\
(\text { mean } \pm \mathrm{SD}), \%\end{array}$ & $\begin{array}{l}\text { Significant pairwise } \\
\text { comparisons between } \\
\text { subportions }(\mathrm{p}<0.05)\end{array}$ \\
\hline Superficial & $\mathrm{a}$ & $9.5 \pm 5.4$ & $\mathrm{~b}, \mathrm{c}, \mathrm{d}$ \\
masseter & $\mathrm{b}$ & $2.5 \pm 1.9$ & $\mathrm{a}, \mathrm{e}, \mathrm{f}, \mathrm{g}, \mathrm{h}$ \\
& $\mathrm{c}$ & $3.4 \pm 2.7$ & $\mathrm{a}, \mathrm{f}, \mathrm{g}, \mathrm{h}$ \\
& $\mathrm{d}$ & $3.5 \pm 3.0$ & $\mathrm{a}, \mathrm{e}, \mathrm{f}, \mathrm{g}, \mathrm{h}$ \\
\hline Deep & $\mathrm{e}$ & $12.2 \pm 3.2$ & $\mathrm{~b}, \mathrm{c}, \mathrm{d}, \mathrm{f}$ \\
masseter & $\mathrm{f}$ & $8.0 \pm 2.3$ & $\mathrm{~b}, \mathrm{c}, \mathrm{d}, \mathrm{e}$ \\
& $\mathrm{g}$ & $9.1 \pm 3.5$ & $\mathrm{~b}, \mathrm{c}, \mathrm{d}, \mathrm{e}$ \\
& $\mathrm{h}$ & $9.6 \pm 3.6$ & $\mathrm{~b}, \mathrm{c}, \mathrm{d}, \mathrm{e}$ \\
& &
\end{tabular}

Fig. 2. Identification of the regions of interest. a Two points were firstly selected: the zygomatic point (Zyg), as the most inferior point midway along the zygomatic arch; and the gonion (Go) point as the point where the lower border of the mandible curves upward. The original coronal MRI slices were reoriented according to the zygomatic and gonion points. The reoriented coronal MRI slice passing through the zygomatic and gonion points was considered as the coronal reference plane. The cross-sectional plane perpendicular to the coronal reference plane and passing through the midpoint of the distance gonion point - zygomatic point was considered as the axial reference plane. The sagittal plane, perpendicular to the other reference planes, and passing through zygomatic and gonion points, was considered as the sagittal reference plane. Eight masseter muscle subvolumes were then identified. $\mathbf{b}$ The table shows the mean values and standard deviations of the aponeurosis relative content in each masseter subvolume. Aponeurosis relative content was calculated by dividing the aponeuroses volume by muscle volume times 100 . Post hoc multiple comparisons were corrected by the Bonferroni method. Note the relatively high aponeurosis content of the anterior-cranial subvolume, and deep masseter subvolumes. ented according to the zygomatic and gonion points, providing the coronal reference plane. The cross-sectional plane perpendicular to the coronal reference plane and passing through the midpoint of the distance gonion point - zygomatic point was the axial reference plane. The sagittal reference plane was chosen perpendicular to the other reference planes, and passing through the zygomatic and gonion points. Eight muscle subvolumes were then identified.

The volume of masseter aponeuroses for each masseter subvolume was assessed via software. In order to estimate the method error, muscle and aponeurosis volumes were assessed twice in a subset of 6 participants, with at least 1 week interval between measurements.

\section{Statistics}

Preliminary analyses consisted of descriptive statistics, normality tests and tests for homogeneity of variances. Independent Student's $\mathrm{t}$ tests were used to test gender differences in body height, weight and BMI.

The quantitative outcome measurements obtained from each masseter were muscle volume, aponeurosis volume and aponeurosis relative content (i.e. aponeurosis volume divided by muscle volume times 100). The outcome measures were analyzed by means of repeated-measures analysis of variance, using muscle subvolume (eight levels) as within-subject factor, and gender as between-subject factor. Age, body height and body weight were 


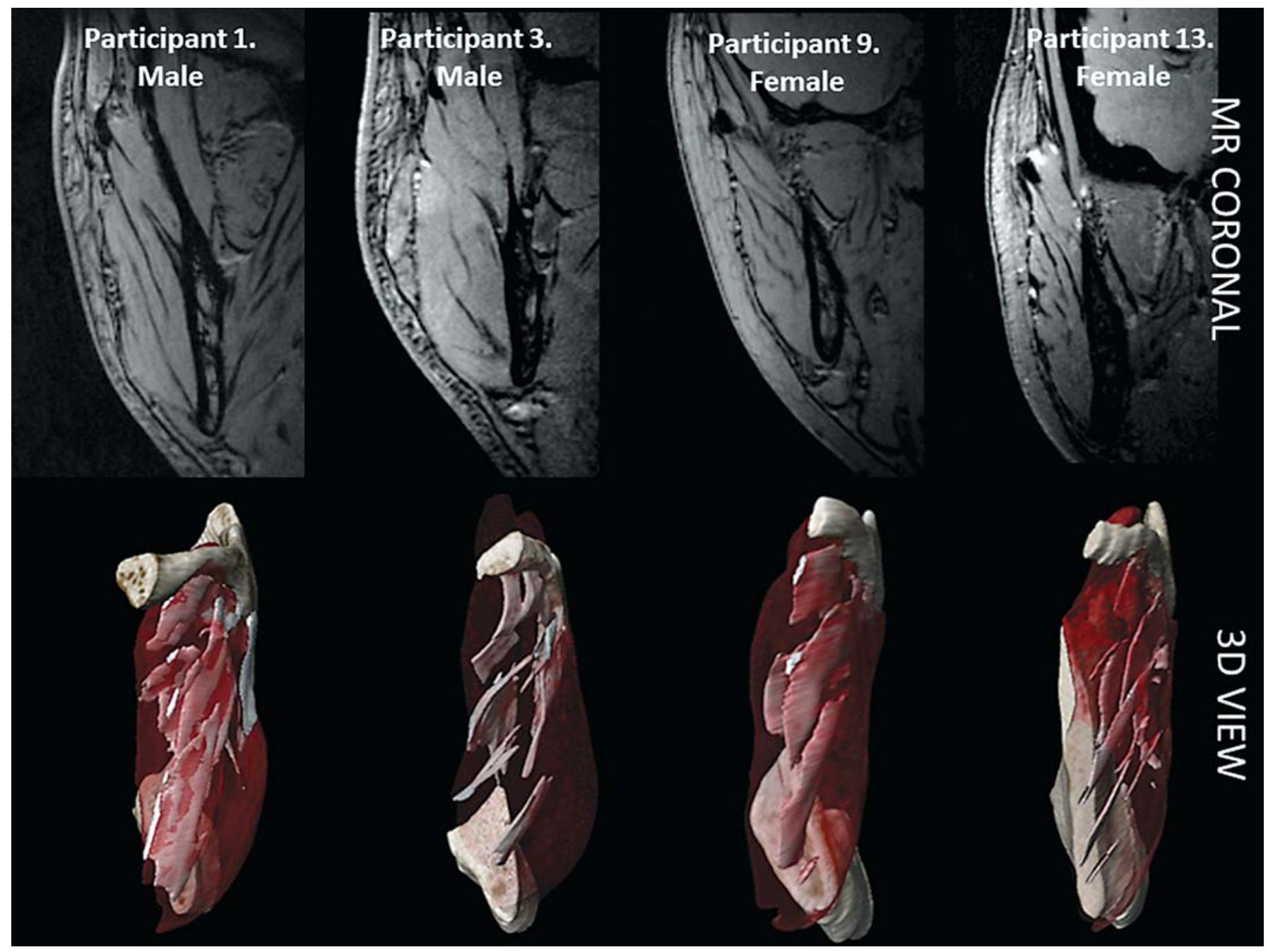

Fig. 3. Four examples of MRI coronal scans with their corresponding three-dimensional rendering of the masseter muscles illustrating the internal architecture of their aponeuroses. Each masseter muscle is shown from a posterior view. Note the marked interindividual variability. The complete set of high-resolution images is given in online supplementary figures 1-14.

entered into the model as continuous covariates. Correlations between masseter volume, aponeurosis volume, body weight and body height were computed by Pearson coefficients. All the analyses were performed using commercial software (SPSS 15.0, Chicago, Ill., USA). Type I error was set at $\mathrm{p} \leq 0.05$ (two-tailed).

\section{Results}

The mean \pm SD body height of the male and female subjects was $177.5 \pm 6.1 \mathrm{~cm}$ and $166.2 \pm 4.9 \mathrm{~cm}$, respectively. The mean \pm SD body weight was $76.3 \pm 9.3 \mathrm{~kg}$ in males and $59.6 \pm 5.4 \mathrm{~kg}$ in females. BMI was $24.2 \pm 2.6$ in males and $21.6 \pm 2.4$ in females. Males and females differed significantly for body height $(\mathrm{p}=0.002)$, body weight ( $p=0.001)$ but not for BMIs $(\mathrm{p}=0.074)$.

The mean \pm SD volume of masseter muscle was 28.8 $\pm 7.7 \mathrm{~cm}^{3}$ and was significantly influenced by gender $(\mathrm{F}=22.6 ; \mathrm{p}<0.001)$, being lower in females $(22.0 \pm 5.9$ $\left.\mathrm{cm}^{3}\right)$ than in males $\left(33.4 \pm 3.0 \mathrm{~cm}^{3}\right)$. This difference, however, was not statistically significant $(\mathrm{F}=4.5 ; \mathrm{p}=$ 0.06) after adjusting for body height and body weight.

Individual high-resolution three-dimensional images of the masseter muscles are given as online supplementary figures 1-14 (www.karger.com/doi/10.1159/000329503). Thick aponeuroses were present in all the investigated 
muscles. They generally assumed a fan-shape distribution originating from the zygomatic arch and delimited a number of different muscle compartments (fig. 3). The threedimensional arrangement of these compartments was rather complex and could not be univocally described, as each subject differed with respect to their number, size and locations (for descriptive details, see online suppl. fig. 1-14).

The mean \pm SD volume of masseter aponeuroses was $2.0 \pm 0.8 \mathrm{~cm}^{3}$, and was significantly influenced by gender $(\mathrm{F}=7.4 ; \mathrm{p}=0.018)$ amounting to $1.5 \pm 0.6 \mathrm{~cm}^{3}$ in females, and to $2.5 \pm 0.7 \mathrm{~cm}^{3}$ in males. However, the difference was not statistically significant after adjusting the model for the effect of body height and body weight $(\mathrm{F}=3.0 ; \mathrm{p}=0.08)$.

The mean \pm SD relative content of masseter aponeurosis was $7.1 \pm 2.1 \%$, but it varied significantly across the different masseter subvolumes $(F=21.9 ; p<0.001)$, with the highest content in the most anterior and cranial subvolumes. There were more aponeuroses in the deep than in the superficial masseter muscle subvolumes (fig. 3b).

Masseter volume was significantly correlated with aponeurosis volume $(\mathrm{r}=0.75 ; \mathrm{p}=0.002)$, body height $(\mathrm{r}=0.56$; $\mathrm{p}=0.03)$ and body weight $(\mathrm{r}=0.75 ; \mathrm{p}=0.002)$. Aponeurosis volume was significantly correlated with body height $(\mathrm{r}=0.56 ; \mathrm{p}=0.04)$ and body weight $(\mathrm{r}=0.66 ; \mathrm{p}=0.01)$.

The method error for duplicate measurement of muscle and aponeurosis volume was generally small as it ranged from 0.45 to $1.5 \mathrm{~cm}^{3}(1.5-5.3 \%)$ for masseter volume and from 0.06 to $0.14 \mathrm{~cm}^{3}(3.0-6.8 \%)$ for aponeurosis volume.

\section{Discussion}

Although the human masseter is generally described as a compartmentalized muscle [Eriksson and Thornell, 1983; Van Eijden and Turkawski, 2001; Widmer et al., 2007], only few studies have investigated its internal architecture [Schumacher, 1961; Lam et al., 1991; Minowa et al., 1998; Gaudy et al., 2000]. This study showed that the masseter muscle was not divided into specific muscle compartments by the aponeuroses in a consistent fashion as reported by previous studies of which three distinguished anatomical layers (superficial, intermediate and deep) delimiting 3-5 clear-cut muscle compartments [Schumacher, 1961; Gaudy et al., 2000]. Indeed each investigated subject showed a peculiar and complex three-dimensional masseter compartmentalization. This marked interindividual anatomical variability may represent the neuroanatomical substrate for the widely re- ported functional heterogeneity of the masseter and for the previous observations that a specific jaw motor task can be performed using a multitude of neuromuscular control strategies [Blanksma et al., 1992; Blanksma and Van Eijden, 1995; Farella et al., 2009]. Heterogeneous contractions of the masseter muscles during functional and nonfunctional jaw tasks as well as during prolonged low-level contractions have recently been found by using high-density EMG recordings [Palla and Farella, 2010]. This method relies on multielectrode grids, which are used to describe the two-dimensional spatial distribution of muscle activity (i.e. activity map) and has been used in a variety of muscles [Drost et al., 2006], including the masticatory muscles [Schumann et al., 1994].

The aponeurosis content varied systematically across different muscle subvolumes, being denser in the anterior-cranial portions close to the zygomatic arch and in the deep masseter subvolumes. In agreement with other findings [Gaudy et al., 2010], muscle aponeuroses exhibit a fan shape arrangement in both antero-posterior and mediolateral directions. Understanding the internal arrangement of masseter muscle aponeuroses may be very useful for the development of a three-dimensional navigation system, which allows the stereotactic implantation of EMG electrodes within specific compartments of the masseter muscle [McMillan and Hannam, 1989; Tonndorf et al., 1994]. A real-time MRI-based navigation system for the masseter muscle is currently being developed at the University of Zürich.

The mean values of masseter volume resulting from our study is consistent with previous findings [Dheyriat et al., 1997; Benington et al., 1999; Boom et al., 2008], and similarly to these studies, masseter volume was larger in male than in female participants. Differences in muscle volume can probably be ascribed to gender-related hormonal factors [Bardin and Catterall, 1981]. Also the volume of masseter aponeuroses differed significantly between male and female participants. To the best of our knowledge, this finding has not been previously reported and may have some clinical implications. Indeed, aponeuroses contribute to the storage of elastic energy and play an important role in protecting muscle fibers from contraction-induced or high-strain injuries [Griffiths, 1991]. Thus, the reduced amount and volume of masseter aponeuroses in females might represent a risk factor for postexercise masticatory muscle pain [Plesh et al., 1998].

It can be argued that males and females were not matched for body weight and height. However, in order to control for those factors, the regression model was adjusted for body height and weight. With this adjustment, 
the gender differences in both muscle and aponeurosis volumes disappeared although a tendency $(0.05 \leq \mathrm{p} \leq$ 0.1 ) towards statistical significance was still present. Further studies with greater sample size may help clarify this relationship.

This study has a number of limitations. First, our MRI findings were not validated using histology as reference standard. However, Minowa et al. [1998], who showed in cadavers that MRI is a valid tool to study the inner masseter architecture qualitatively and quantitatively had already tested the feasibility of MRI segmentation to evaluate the aponeurosis content in the human masseter. These authors compared the distribution and thickness of aponeuroses as determined by means of MRI with those obtained from histological sections.

It might be argued that other noninvasive imaging techniques, such as three-dimensional ultrasonography and high-resolution computed tomography, could be used to describe the internal compartmentalization and the aponeuroses of the masseter muscle with a quality similar to or even superior to MRI [Kubo et al., 2006]. Nevertheless, ultrasonography is very sensitive to operator- and technique-related factors [Bertram et al., 2003] and the quality of the images can be influenced by the pressure exerted when holding the probe [Serra et al., 2008]. This pressure may easily interfere with the shape of muscle aponeuroses, which are soft and can be easily deformed. Computed tomography may represent a viable alternative; however, it is associated with a biological risk due to radiation, and is, therefore, not suitable to investigate the internal masseter architecture in healthy subjects.

The quality of MR images was satisfactory in all subjects and although time-consuming, it allowed easy identification of muscle boundaries and aponeuroses. The segmentation was primarily performed using coronal MR slices. However, the other ones (axial and sagittal), obtained by reformatting the coronal ones, were used if necessary to better identify the boundaries of muscle and aponeuroses. A significant limitation of our approach is that, due to resolution constraints, it was difficult to accurately detect the thinnest aponeuroses (e.g. below 0.5 $\mathrm{mm}$ ), which could even have been missed from segmentation. This means that the volume of masseter aponeuroses resulting from this study may have been somewhat underestimated. Three-dimensional rendering of anatomical structures by MRI, however, has the undisputed advantage of being noninvasive and sufficiently accurate [Goebel et al., 2010].

The aim of this study was to get an insight in the threedimensional anatomy of the masseter muscle compart- ments and not to provide data for biomechanical analyses. This was done in order to possibly obtain a template of the internal architecture of the masseter which could be used in EMG studies on the activation patterns of this muscle during different tasks or prolonged contractions. Therefore, the volume rather than the cross-section of the masseter muscle and aponeurotic tissue was analyzed. Of course, from a biomechanical point of view, the most characteristic structural parameter for any tissue under longitudinal stress is its cross-section rather than its volume. In order to address some biomechanically relevant anatomical issues, further MRI analyses are needed. For instance, it may be interesting to investigate whether this technique may help depicting the orientation of the collagen fibers in relationship to the muscle fibers; this is an important issue because the effect of muscle fibers on aponeurosis strain depends upon the fiber pennation angle [Chi et al., 2010].

Other limitations of the current study were the choice of a convenience sample, which included healthy students or staff member of the University of Zürich, and of the right masseter for analysis. Hence, the study participants may be not representative of the general population, and the possible occurrence of a selection bias cannot be excluded. One-side analysis prevents inferring on possible side differences in the masseter architecture. This is a significant limitation of the study, especially considering that patients with myogenous craniomandibular disorders usually have unilateral pain, and that when pain is bilateral, it is more severe on one side. The choice to analyze only one side was dictated by the study feasibility as the complete segmentation of each masseter muscle took several days.

In conclusion, our findings confirmed that the masseter is a compartmentalized muscle, but it also indicated that its internal architecture varies markedly across individuals. The aponeuroses were largely represented within the masseter, and their content varied systematically across different masseter subportions. Gender-related differences were found for both muscle and aponeurosis volumes, but they disappeared after adjusting for body weight and height.

\section{Acknowledgements}

This study was supported by a grant from the Swiss National Science Foundation (SNF-320000-120592). A special thanks to all the volunteers who participated in this study. This work was carried out at the Clinic for Masticatory Disorders, Removable Prosthodontics and Special Care Dentistry, University of Zürich, Switzerland. 


\section{References}

Augat P., F. Eckstein (2008) Quantitative imaging of musculoskeletal tissue. Annu Rev Biomed Eng 10: 369-390.

Bardin C.W., J.F. Catterall (1981) Testosterone: a major determinant of extragenital sexual dimorphism. Science 211: 1285-1294.

Benington P.C., J.E. Gardener, N.P. Hunt (1999) Masseter muscle volume measured using ultrasonography and its relationship with facial morphology. Eur J Orthod 21: 659-670.

Bertram S., I. Brandlmaier, A. Rudisch, G. Bodner, R. Emshoff (2003) Cross-sectional characteristics of the masseter muscle: an ultrasonographic study. Int J Oral Maxillofac Surg 32: 64-68.

Blanksma N.G., T.M. Van Eijden (1995) Electromyographic heterogeneity in the human temporalis and masseter muscles during static biting, open/close excursions, and chewing. J Dent Res 74: 1318-1327.

Blanksma N.G., T.M. Van Eijden, W.A. Weijs (1992) Electromyographic heterogeneity in the human masseter muscle. J Dent Res 71: 47-52.

Boom H.P., P.H. van Spronsen, F.C. van Ginkel, R.A. van Schijndel, J.A. Castelijns, D.B. Tuinzing (2008) A comparison of human jaw muscle cross-sectional area and volume in long- and short-face subjects, using MRI. Arch Oral Biol 53: 273-281.

Chi S.W., J. Hodgson, J.S. Chen, V. Reggie Edgerton, D.D. Shin, R.A. Roiz, S. Sinha (2010) Finite element modeling reveals complex strain mechanics in the aponeuroses of contracting skeletal muscle. J Biomech 43: 1243 1250.

Dheyriat A., D. Sappey-Marinier, M. Lissac, A Bonmartin (1997) Anatomical study with MRI of a small muscle: the masseter. J Radiol 78: 359-366.

Drost G., D.F. Stegeman, B.G. van Engelen, M.J Zwarts (2006) Clinical applications of highdensity surface EMG: a systematic review. J Electromyogr Kinesiol 16: 586-602.

Dworkin S.F., L. Le Resche (1992) Research diagnostic criteria for temporomandibular disorders: review, criteria, examinations and specifications, critique. J Craniomandib Disord 6: 301-355.

Eriksson P.O., L.E. Thornell (1983) Histochemical and morphological muscle-fibre characteristics of the human masseter, the medial pterygoid and the temporal muscles. Arch Oral Biol 28: 781-795.

Falla D., H. Andersen, B. Danneskiold-Samsoe, L. Arendt-Nielsen, D. Farina (2010) Adaptations of upper trapezius muscle activity during sustained contractions in women with fibromyalgia. J Electromyogr Kinesiol 20: 457-464.

Falla D., D. Farina (2008) Non-uniform adaptation of motor unit discharge rates during sustained static contraction of the upper trapezius muscle. Exp Brain Res 191: 363-370.
Farella M., S. Palla, S. Erni, A. Michelotti, L.M. Gallo (2008) Masticatory muscle activity during deliberately performed oral tasks. Physiol Meas 29: 1397-1410.

Farella M., A. Palumbo, S. Milani, S. Avecone, L.M. Gallo, A. Michelotti (2009) Synergist coactivation and substitution pattern of the human masseter and temporalis muscles during sustained static contractions. Clin Neurophysiol 120: 190-197.

Farina D, F. Leclerc, L. Arendt-Nielsen, O. Buttelli, P. Madeleine (2008) The change in spatial distribution of upper trapezius muscle activity is correlated to contraction duration. J Electromyogr Kinesiol 18: 16-25.

Gaudy J.F., A. Zouaoui, P. Bravetti, J.L. Charrier, A. Guettaf (2000) Functional organization of the human masseter muscle. Surg Radiol Anat 22: 181-90.

Goebel J.C., A. Pinzano, D. Grenier, A.L. Perrier, C. Henrionnet, L. Galois, P. Gillet, O. Beuf (2010) New trends in MRI of cartilage: advances and limitations in small animal studies. Biomed Mater Eng 20: 189-194.

Griffiths R.I. (1991) Shortening of muscle fibres during stretch of the active cat medial gastrocnemius muscle: the role of tendon compliance. J Physiol 436: 219-236.

Hägg G.M. (1991) Static work loads and occupational myalgia - a new explanation model; in Anderson, P.A., D.J. Hobart, J.V. Danoff (eds): Electromyographical Kinesiology. Amsterdam, Elsevier, pp 141-144.

Huang G.J., L. LeResche, C.W. Critchlow, M.D. Martin, M.T. Drangsholt (2002) Risk factors for diagnostic subgroups of painful temporomandibular disorders (TMD). J Dent Res 81: 284-288.

Koolstra J.H., T.M. Van Eijden (1992) Application and validation of a three-dimensional mathematical model of the human masticatory system in vivo. J Biomech 25: 175-187.

Kubo K., T. Kawata, T. Ogawa, M. Watanabe, Sasaki K (2006) Outer shape changes of human masseter with contraction by ultrasound morphometry. Arch Oral Biol 51: 146-153.

Lam E.W., A.G. Hannam, E.L. Christiansen (1991) Estimation of tendon-plane orientation within human masseter muscle from reconstructed magnetic resonance images. Arch Oral Biol 36: 845-853.

McMillan A.S. (1993) Task-related behaviour of motor units in the human temporalis muscle. Exp Brain Res 94: 336-342.

McMillan A.S., A.G. Hannam (1989) Location of needle electrode recording sites in the human masseter muscle by magnetic resonance imaging. J Neurosci Methods 30: 85-89.

McMillan A.S., A.G. Hannam (1992) Task-related behavior of motor units in different regions of the human masseter muscle. Arch Oral Biol 37: 849-857.
Michelotti A., I. Cioffi, P. Festa, G. Scala, M. Farella (2010) Oral parafunctions as risk factors for diagnostic TMD subgroups. J Oral Rehabil 37: 157-162.

Minowa K., N. Inoue, Y. Ashikaga, S. Yoshida, Y. Totsuka, M. Nakamura (1998) Comparison of magnetic resonance imaging and gross findings regarding masseter muscle aponeuroses in cadavers. Oral Surg Oral Med Oral Pathol Oral Radiol Endod 86: 275-279.

Ng H.P., S.H. Ong, K.W. Foong, P.S. Goh, W.L. Nowinski (2006) Knowledge-driven 3-D extraction of the masseter from MR data. Conf Proc IEEE Eng Med Biol Soc 1: 5294-5297.

Ng H.P., S.H. Ong, K.W. Foong, P.S. Goh, W.L. Nowinski (2008) Masseter segmentation using an improved watershed algorithm with unsupervised classification. Comput Biol Med 38: 171-184.

Niioka T., H. Ishii, H. Izumi (2009) Regional differences in blood flow variation in rat masseter muscle. Arch Oral Biol 54: 1022-1028.

Palla S., M. Farella (2010). Masticatory muscle pain; in Mense S., R.D. Gerwin (eds): Muscle Pain: Diagnosis and Treatment. Springer, Berlin, pp 193-227.

Plesh O., D.A. Curtis, L.J. Hall, A. Miller (1998) Gender difference in jaw pain induced by clenching. J Oral Rehabil 25: 258-263.

Schindler H.J., J.C. Türp, R. Blaser, J. Lenz (2005) Differential activity patterns in the masseter muscle under simulated clenching and grinding forces. J Oral Rehabil 32: 552-563.

Schumacher G.H. (1961) Funktionelle Morphologie der Kaumuskulatur. Berlin, Fischer Verlag.

Schumann N.P., H.C. Scholle, C. Anders, E. Mey (1994) A topographical analysis of spectral electromyographic data of the human masseter muscle under different functional conditions in healthy subjects. Arch Oral Biol 39: 369-377.

Serra M.D., M.B. Duarte Gavião, M.N. dos Santos Uchôa (2008) The use of ultrasound in the investigation of the muscles of mastication. Ultrasound Med Biol 34: 1875-1884.

Sjøgaard G., K. Søgaard (1998) Muscle injury in repetitive motion disorders. Clin Orthop Relat Res 351: 21-31.

Tonndorf M.L., D.G. Connell, A.G. Hannam (1994) Stereotactic location of EMG needle electrode scans relative to tendons in the human masseter muscle. J Neurosci Methods 51: 77-82.

Van Eijden T.M., S.J. Turkawski (2001) Morphology and physiology of masticatory muscle motor units. Crit Rev Oral Biol Med 12: 7691.

Widmer C.G., A.W. English, J. Morris-Wiman (2007) Developmental and functional considerations of masseter muscle partitioning. Arch Oral Biol 52: 305-308 